\title{
USE OF ELASTOGRAPHY FOR CERVICAL CANCER DIAGNOSTICS
}

\author{
O.A. Bakay*, T.S. Golovko \\ National Cancer Institute, Kyiv 03022, Ukraine
}

\begin{abstract}
The aim of the study was to investigate the use of elastography for cervical cancer diagnostics. Materials and Methods: The ultrasonic study with use of sonoelastography involved 87 patients with cervical pathology (cervicitis, $n=11$; dysplasia, $n=14$; cervical cancer, $n=62$ ). Results: At non-malignant pathology elastic types of elastograms prevailed while the tumors were characterized by images of stiff type formations. Present study has demonstrated an effectiveness of sonoelastography for valuation of tumor process invasion levels. At detection of tumor invasion into parametrium, the test sensitivity increased from 77.1 to $91.4 \%$, specificity - from 85.1 to $96.2 \%$, accuracy — from 80.6 to $93.5 \%$; in case of tumor invasion into vagina these parameters correspondently changed: from 75.0 to $83.3 \%$, from 76.9 to $88.4 \%$, from 75.8 to $85.4 \%$, respectively; and in case of uterus: from 82.9 to $85.3 \%$, from 85.7 to $95.2 \%$, from 83.8 to $88.7 \%$, respectively. Conclusions: Inclusion of elastography into ultrasound examination of cervical pathologies could significantly improve diagnostic quality of the method.
\end{abstract}

Key Words: sonoelastography, ultrasonic diagnostics, cervical cancer.

Significant increase of cervical cancer (CC) incidence stipulates necessity of this pathology diagnostic methods improvement and new technologies development. At present, new method of ultrasonic investigation (USI) - sonoelastography estimating tissues elasticity becomes more and more popular. In opinion of many researchers it is a very useful additional method for diagnostics of differently localized pathologies [1, 2].

It is known that elasticity is one of the most important tissues characteristics, especially valuable for a diagnostician, as it is changing at different pathologic processes (alterations, tumors, traumas, etc.). Any new formations with high stiffness are associated with increased risk of malignancy [3].

Elastography is based on the same principle as palpation. The pressure causes deformation of tissues, more soft tissues would be deformed more profoundly then more stiff ones. Valuation of this deformation rates allows obtaining information on tissues elasticity. At elastographic investigation pressure is created by ultrasonic wave or by light mechanic compression. Ultrasonic detection allows estimating different tissues deformations due to created pressure. Obtained data are analyzed by computer software for echo signal processing and elastogram producing where different elasticity values are marked with different colors. Sometimes elastography is called "method of visual palpation" [4].

Thought elastography development started at the end of 90's, at present are only few publications on this method possibilities at the field of gynecology [5-8]. Elastography use at oncogynecology for cervix pathologies diagnostics at present remains insufficiently studied.

The aim of our present study has been investigation of elastography possibilities for cervix tumors diagnostics.

\footnotetext{
Submitted: January 28, 2015.

*Correspondence: Tel.: +380503557442

E-mail: bvgolga@mail.ru
}

Abbreviations used: CC - cervical cancer; EG - elastography;

MRI - magnetic resonance imaging; USI - ultrasonic investigation.

\section{MATERIALS AND METHODS}

The study involved 87 patients with cervical pathology in age from 28 to 61 years. The median age was 46.5 years. In 11 cases was detected cervicitis, in 14 cases - dysplasia, in 62-CC. CC stages distribution pattern was as follows: cancer in situ $-4(6.5 \%)$ patients, la stage $-5(8.1 \%)$, lb stage $-9(14.5 \%)$, $\mathrm{Ila}-9$ (14.5\%), Illb -8 (12.9\%), Illa -14 (22.6\%), IIIb $-11(17.7 \%)$, IVa -2 (3.2\%). CC morphologic structures of all patients were verified. Thus in $34(54.8 \%)$ patients squamous cell carcinoma at different stages of differentiation was diagnosed, in $12(19.4 \%)$ patients - tumors of androgen origin were detected, in $6(9.7 \%)$ patients - other histological forms of carcinoma (clear cell, small cell etc.), in $10(16.1 \%)$ patients - undifferentiated tumors. Surgically treated were 26 women (among them: cone resection -8 patients with cancer in situ and la stage, trachelectomy -2 patients with CC Ib stage, hysterectomy -16 patients with CC la-lla stages). 48 patients with CC IIb-IV stages obtained chemoradiotherapy. In 4 patients, with treated CC, relapse of tumor was detected. Control group included 30 healthy women ( 18 of reproductive age and 12 - in menopausa).

Complex USI with use of elastography were conducted for all patients at Esaote MyLab Class C device, Italy, with multi-frequency wideband sensors: Convex CA541, transvaginal EC1123 and linear LA523. Investigations were carried out as follows. Firstly - standard transabdominal and transvaginal USI (B-mode Doppler). After activation elastography regime region of interest was included into the interrogation zone.

All works have been carried out according to device producers manual.

Region of interest parameters must be optimized. It should be noted that the larger would be interrogation zone, the lower spatial resolution we obtain, and vice versa. On elastogram quality influence depth of object location and its mobility (elastography could be the most informative for diagnostics of immobile objects localized on the depth from 0.5 to $5.0 \mathrm{~cm}$ ). 
Sonoelastography regime parameters must be optimized (power, intensifying, color map). The sensor must be held athwart to the surface of the body, without deviations to the right or left, producing lowamplitude rhythmic compression (comparable with a slight tremor), which degree is reflecting at a special display. Sometimes, external compression is not necessary, as if near the area of the survey are large vessels, transfer ripple is enough. The screen shows the elastogram where blue hues mapped tighter areas, and red and green - elastic. If desired color scale can be changed. It is also possible to compare the degree of elasticity of the various areas in the interrogation zone. For this aim on ready elastogram areas of interest must be marked. Then device calculates stiffness ratio in selected areas. It is thus possible to define stiffness differences in area with changes in comparison with unchanged tissues.

Magnetic resonance imaging (MRI) of the 58 patients' pelvic organs was carried out at tomograph Philips (Intera). Ultrasound semiotics was compared with the clinical courses of the diseases, MRI and morphological studies data.

Table 1. Types of elastograms in gynecology

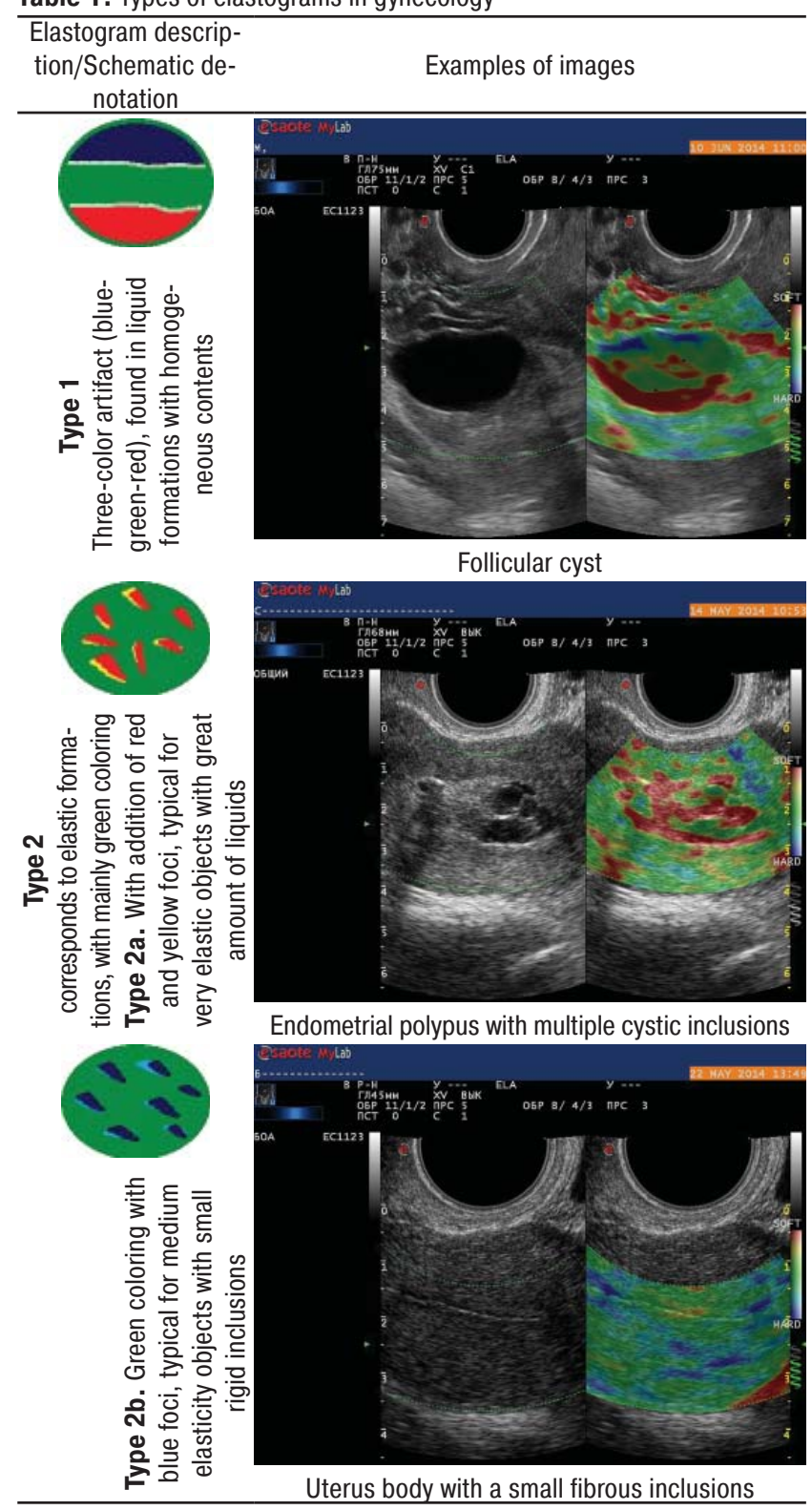

\section{RESULTS}

Elastography results were analyzed and systematized using the classification proposed V. Gazhonova et al. [9], according to which elastic formations could be characterized by colorations types 1 and 2 , while stiff ones - by 3 and 4 (Table 1 ).

As can be seen from the Table 1 , type 1 corresponding to liquid objects, is colored as three-color artifact. In turn, type 2, corresponding to elastic tissues objects, has some variants: 2a characterized very elastic formations, containing great amounts of liquid, $2 \mathrm{~b}$ corresponds to less elastic structures with small amount of small rigid inclusions. Mosaictype, containing both many areas of high compressibility and small rigid inclusions, was classified by us as a variant of the 2 type (2c). The type 3 of elastogram corresponds to stiff formations, with an equal number of areas of high and low compressibility. The type 4 of elastogram is very stiff one usually characteristic for malignant tumors.

During control group patients investigations unchanged cervix has been mapped predominantly green with the addition of blue and red foci (type

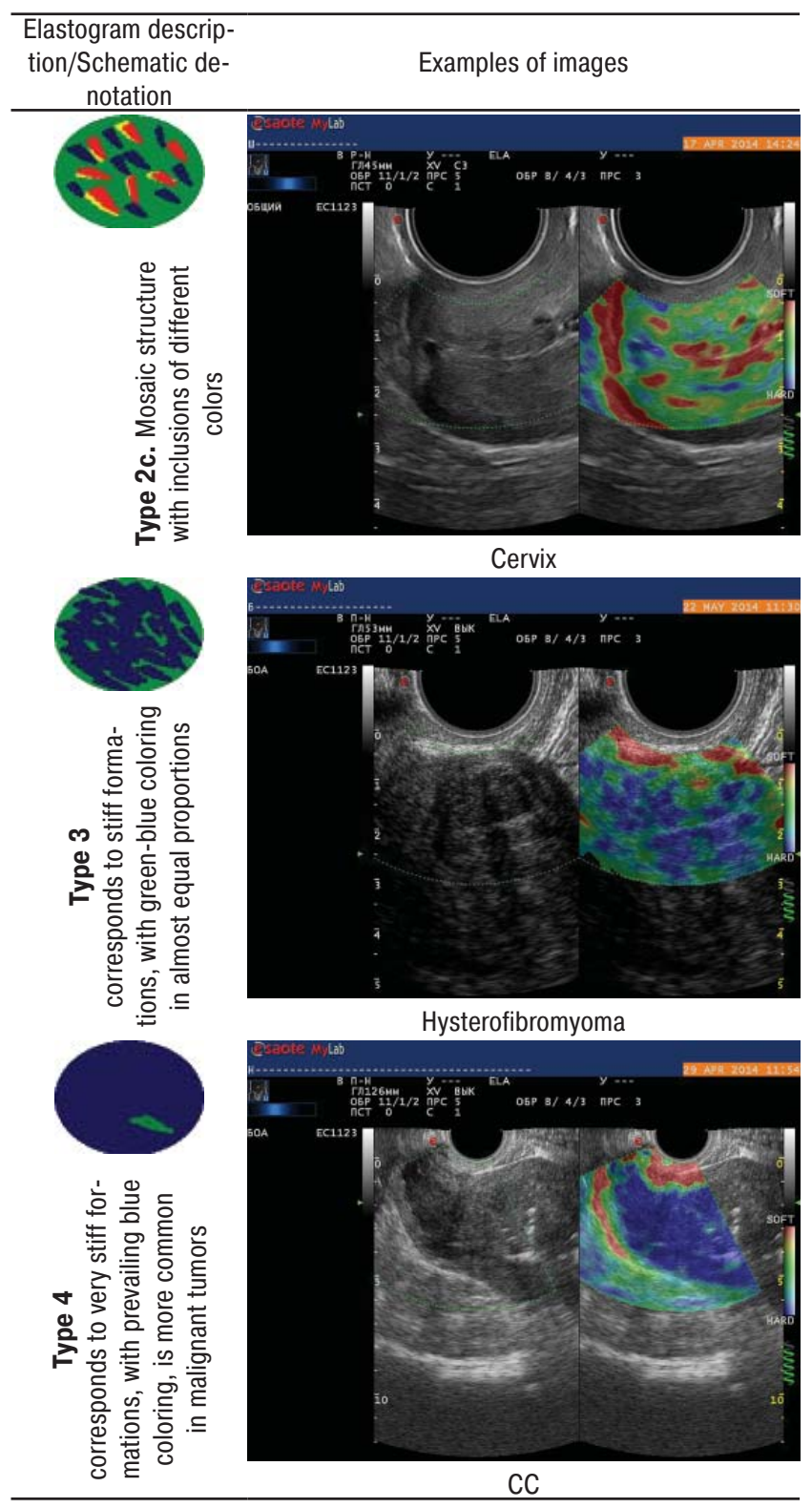


$2 \mathrm{a}, 2 \mathrm{~b}$ and $2 \mathrm{c}$ ). In case of mucus presence in the cervix, it was determined by red coloration along the canal. Small endocervical cysts have been colored by red, the larger ones were of the typical three-color scheme, characteristic for liquid objects. Small blue foci (corresponding to the connective tissue and fibrosis inclusions) were more often defined along the external os and the transformation zone. Outer more looser cervical stroma layer had green coloring. Around the organ was always detected red band corresponding to a very elastic parametrial tissue and to small amounts of mucus in the vaginal vault (Fig. 1).

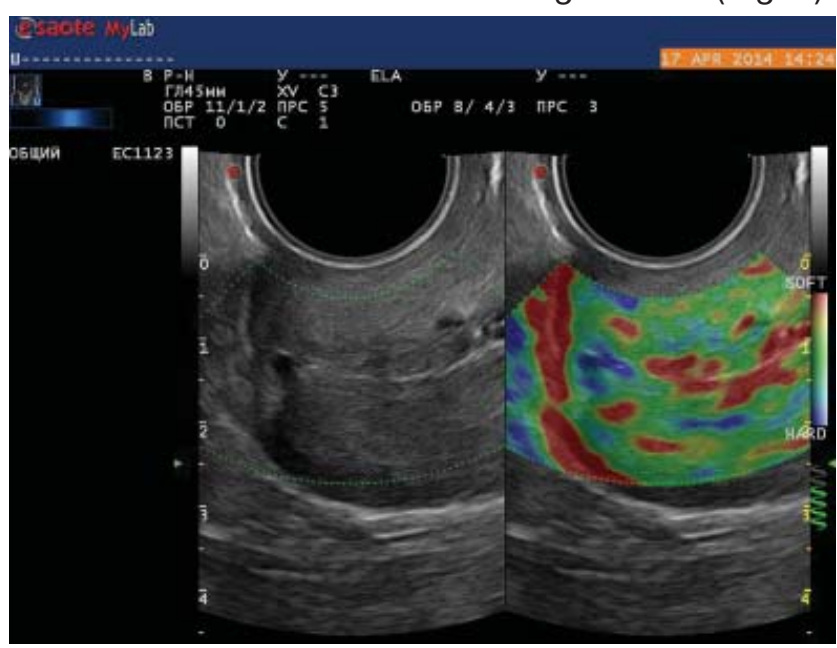

Fig. 1. Normal cervix elastogram at reproductive period

It is known that the cervix tumors at bimanual investigation are dense enough by touch, which allows the clinician to differ them (by palpation) from unmodified elastic stroma [2]. These elasticity differences were also detected at "visual palpation" - elastography.

Cervix with background and precancerous processes (cervicitis, dysplasia) in all cases remained elastic and was colored predominantly by green.

At CC levels of elastographic symptoms manifestations directly depended on tumor sizes and stage of progress, therefore obtained data analysis was carried out in accordance with the tumor process staging in obedience to CC classification by FIGO (2009).

Thus, with cancer in situ we did not detect diagnostically significant differences by elastography investigation.

At CC stage 1a in the majority of cases sonoelastographic images did not practically differ from normal ones, only at $40 \%$ patients with exophytic tumors in the projections of defeats could be seen localized dark blue areas. Taking into account small sizes (up to 5-7 mm) of tumor invasions at this stage, it was difficult to distinguish them from stiff fibrous and connective tissue inclusions as both of them were colored as dark blue foci at the background of unchanged stroma.

Starting from stage $1 \mathrm{~b}$, tumors larger than $1 \mathrm{~cm}$ in all cases had the characteristic images, were mapped as zones of almost solid dark blue color (mostly type 4, sometimes - type 3) on a background of unmodified cervix stroma which allowed to distinguish them reliably from fibrous inclusions and background processes.
Elastography clearly traced tumor contours. In B-mode it was not always easy to trace this border line, as the difference in tints of grey could be so small, that it was difficult to fix it by the eye. Elastography facilitated this process as a dark blue tumor was well distinctive from the green unmodified stroma and red parametrial tissue. The better visualization of tumor contours helped to carry out more accurate measuring and localization of the tumor.

With widespread cancer (II-IV stages), the main tasks of the diagnostic imaging are an estimation of the process distribution levels and precise tumor stages definition which could be of great importance for oncologists, as it radically influences at choice of treatment tactics and final prognosis [10].

CC stage Ila is characterized by the tumor invasion into the uterus and vagina. Unmodified myometrium having the same elasticity as the cervix, is colored in a green with a small amount of blue foci (type $2 b$ ).

In case of tumor invasion into the myometrium, its dark blue image on elastogram has been detected outside of cervix in the body of the uterus, with well traced border between invasion area and elastic myometrium.

Invasion of CC into the vaginal wall in some cases is difficult to differentiate from exophytic tumors protruding into its lumen. At the absence of vagina lesions, its walls are not changed, their surfaces contain small amounts of mucus, which are imaged at elastograms by characteristic red coloring around the external os.

The main feature for distinguishing Ilb from lla stage is the presence of invasion into the parametrial tissue. Its ultrasound visualisation is a rather difficult task. Undoubtedly, devices technical progress and wide distribution of Doppler ultrasound diagnostics greatly improved the possibilities of ultrasound diagnosis in this area [11], but it is necessary to confess that at present $\mathrm{MRI}$ is the most informative for estimating parametrium invasions [12, 13]. Nevertheless, the elastography introduction into a complex ultrasound diagnostics allows us to obtain informing level comparable to MRI.

In our study, we observed correlations between MRI-tomograms and elastograms. Estimation of tumor invasion into parametrium was carried out having regard to the presence of the following sign. In areas where the border of the cervix was preserved, could clearly be seen, green, preserved part of the stroma and the red strip of unaffected paracervical tissue (Fig. 2).

In areas of tumor invasion outside the organ contour was not traced (typical image of cervical stroma and tissues was absent and displaced in projection by multiple dark blue foci of the tumor).

Fig. 3, 4 compares elastograms and MRI-images of patients with the invasions of neoplastic processes into parametrium and the isthmus of the corpus uteri. In case of CC invasion into the bladder, the border with this organ was not traced, red layer between tumor and bladder back wall (consisted of very elastic paravesicular tissue) was absent on elastogram. 

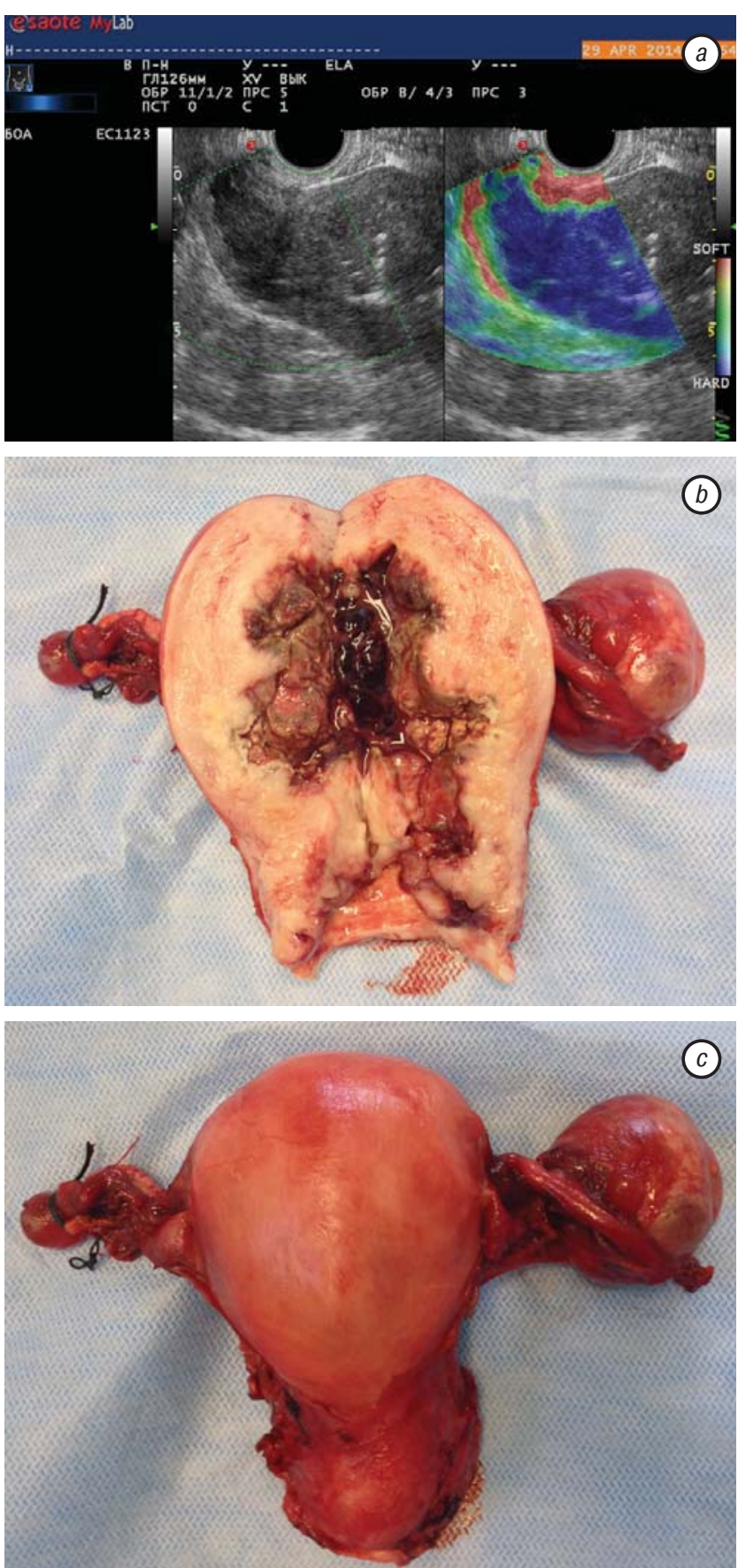

Fig. 2. CC Ila stage. Elastogram of endophytic tumor which significantly affects the cervix stroma and invades uterus body, without leaving its margins. There is a clear border line between dark blue tumor and green-colored unchanged stroma (a). Post-operative photos of tumor confirm the absence of invasion outside the uterus $(b, c)$

It is well known that at CC stages IIb-IV chemoradiotherapy is the main method of treatment [14]. The most important task for diagnostic imaging during such treatment is estimation of its effectiveness at all its phases.

During conservative treatment monitoring standard ultrasound investigations were complemented by elastography. While estimation of tumors' response to treatment a number of parameters were taken into account: changes of sizes, volume, echostructure and character of tumor vascularization, the state of the cervical canal, the extent of tumor invasion into the surrounding tissues, adjacent organs, and the presence of metastases. But the most objective criterion is the degree of tumor volume regression [15]. Usually, this parameter is estimated indirectly by measuring of cervix sizes and volume, which does not allow evaluating state of tumor process objectively enough, as the volume of cancer invasion usually differs from the cervix volume itself.
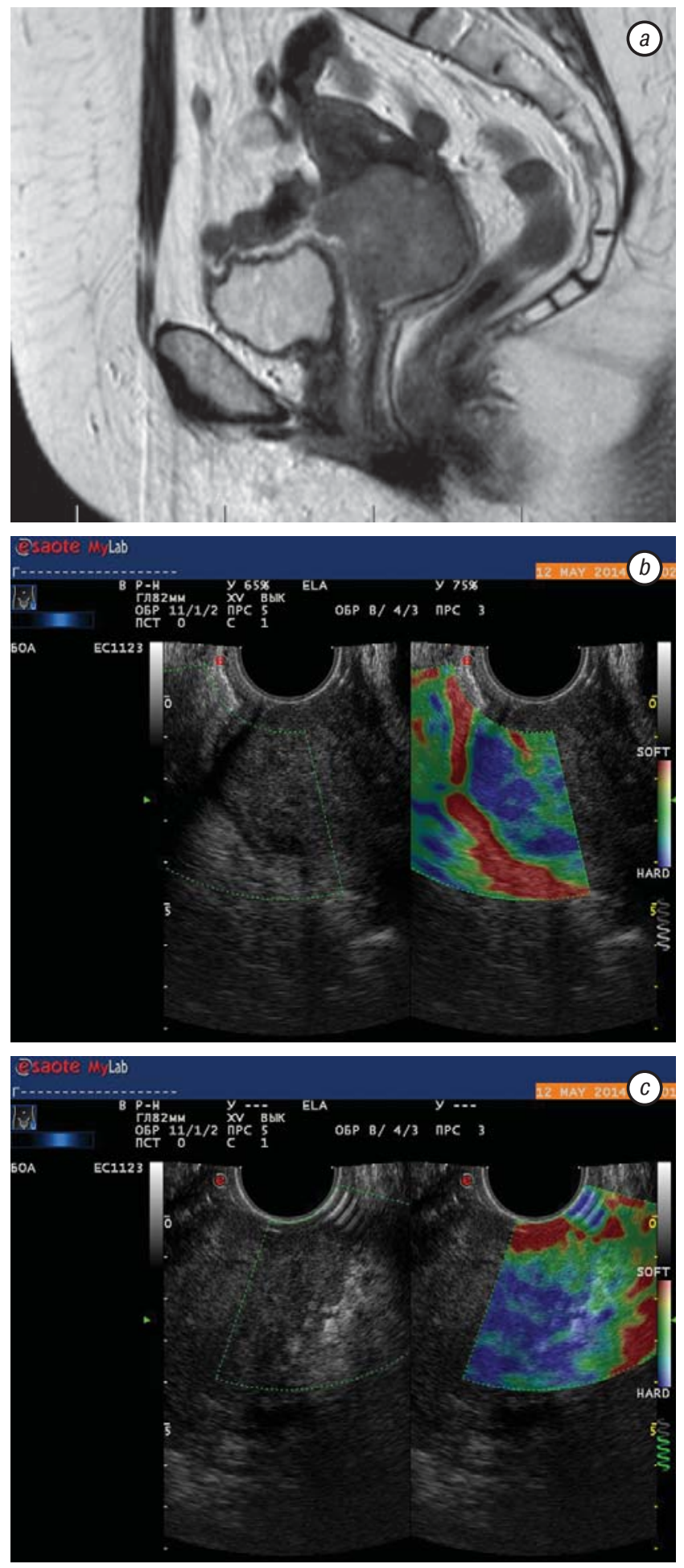

Fig. 3. CC Ilb stage. MRI scan: tumor invasion outside the cervix into the uterus front wall and the body $(a)$; back contour of the cervix is preserved $(b)$, typical image of preserved green stroma and red paracervical tissue is absent at the front side tumor invades outside the organ $(c)$

But direct measuring of tumors' sizes and volumes at broad invasion process is difficult enough, as contours definition during standard ultrasound investigation is often unsimply. Elastography facilitated this task, as improved visualization of tumor contours. Furthermore, after the effective radiotherapy we registered reduction of dark blue 
foci amount, and changes in the tumor stroma stiffness comparatively to intact tissue (Fig. 5).
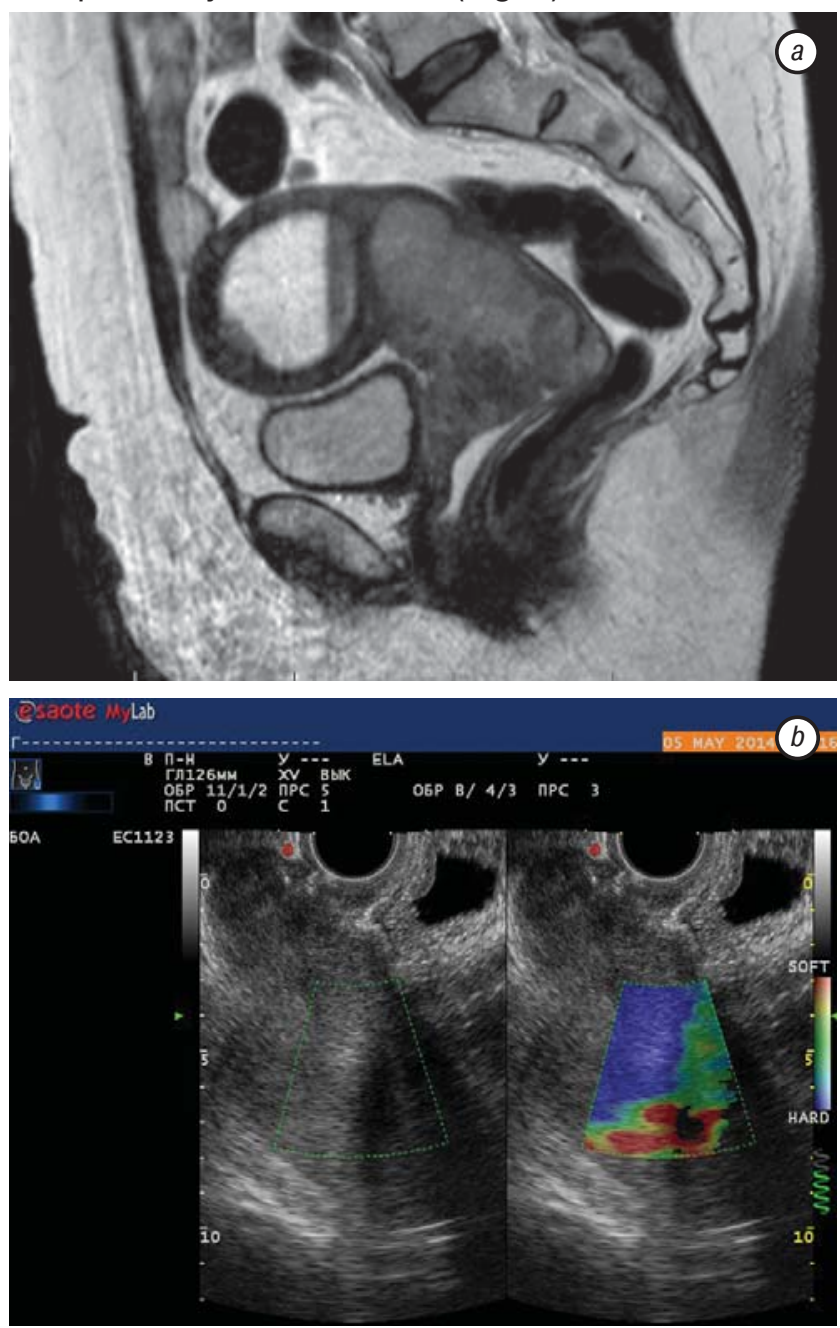

Fig. 4. CC stage III. Tumors' invasion into the isthmus of the corpus uteri: $a-\mathrm{MRl} ; b-$ elastogram

The main task of patients monitoring after conservative treatment finishing is possible recurrence (relapse of disease) early detection [16]. In our investigations in case of its absence cervix sonoelastographic image did not differ from the normal one. However, in general, the number of blue foci were a little greater (predominant type was elastogram $2 b$ ). Such changes corresponded to the post-radiation changes - increased number of although non-malignant but also non-elastic fibrous inclusions.

Elastographic image of CC recurrence corresponded to the image of the primary tumor. The affected area was mapped in dark blue (elastogram type 4). In cases of 2 patients we had to differentiate tumors recurrences from inflammatory changes, which could also cause an increase in organ size, the heterogeneity of the structure and increased vascularization. Inflammatory infiltrate (unlike the tumor) was mapped as elastic formation - mostly green colored (Fig. 6).

\section{DISCUSSION}

Thus, our investigations has been demonstrated that the differences in elasticity of cervix nonmalignant and malignant pathologies are evidently reflected in elastographic images. In non-malignant processes cervix in all cases remained elastic and was mapped predominantly green, while scanning of CC, characterized by a high degree of rigidity, on elastograms prevailed blue coloring. Elastography type's distribution at patients with non-malignant and malignant tumors of the cervix is presented at Fig. 7 .
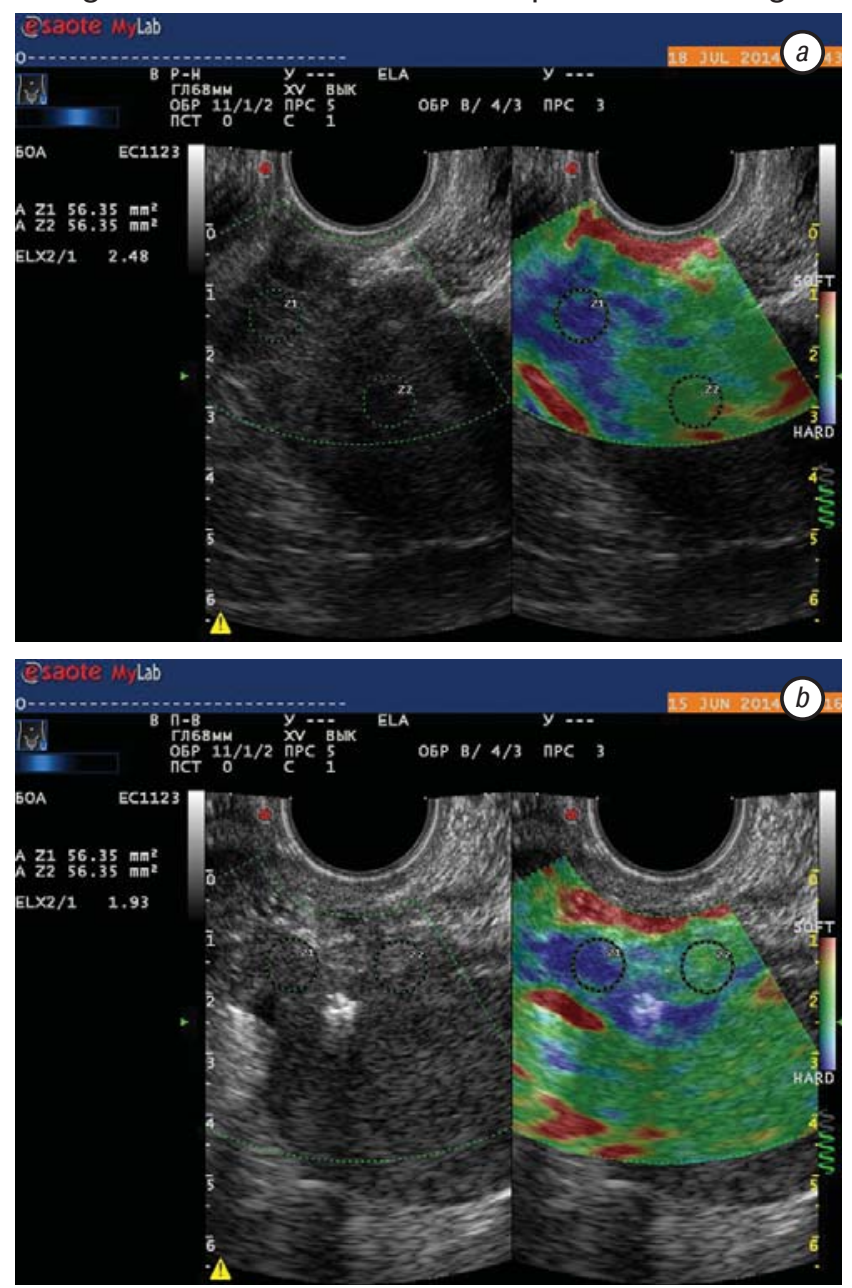

Fig. 5. Monitoring of the chemoradiotherapy effectiveness after stage 1 of radiotherapy (a) and after 2 stage radiotherapy $(b)$ : tumor was reduced in size, number of blue foci lowered as soon as the difference between the stiffness levels in the tumor stroma and intact tissue

As can be seen from the diagram, the stroma of the cervix was mapped by elastogram types 2-4. Only large cysts of the endocervix were colored as elastogram type 1, characteristic for liquid objects. In cases of non-malignant pathologies prevailed elastogram types $2 \mathrm{a}, 2 \mathrm{~b}$ and $2 \mathrm{c}$. In single case of cervical dysplasia we met stiff elastogram type 3, associated with a large number of stroma fibrous inclusions after previuos cone resection. At CC severity of sonoelastographic symptoms manifestation directly depended on the stage of the process. Thus, at the initial invasion (carcinoma in situ, stage la) cervix elastograms images were of elastic types, but just from the lb stage tumors were colored mainly as type $4(75.5 \%)$ and type $3(22.7 \%)$. In cases of advanced cancer presence of green foci at the blue background (type 3) could reflect localization of tumor areas with different levels of stiffness and more elastic regions of necrosis. In the single case of stage IIla $\mathrm{CC}$ we determined mosaic type $2 \mathrm{c}$, which was associated with significant necrosis in tumors, and 
plenty of liquid inclusions (zones of disintegration with a pus). Elastogram type 4 was never met with nonmalignant pathology, but it was typical for CC. Among this it was established, that changes in cervix elasticity, caused by chemo-radiotherapy, were also detected by elastography.
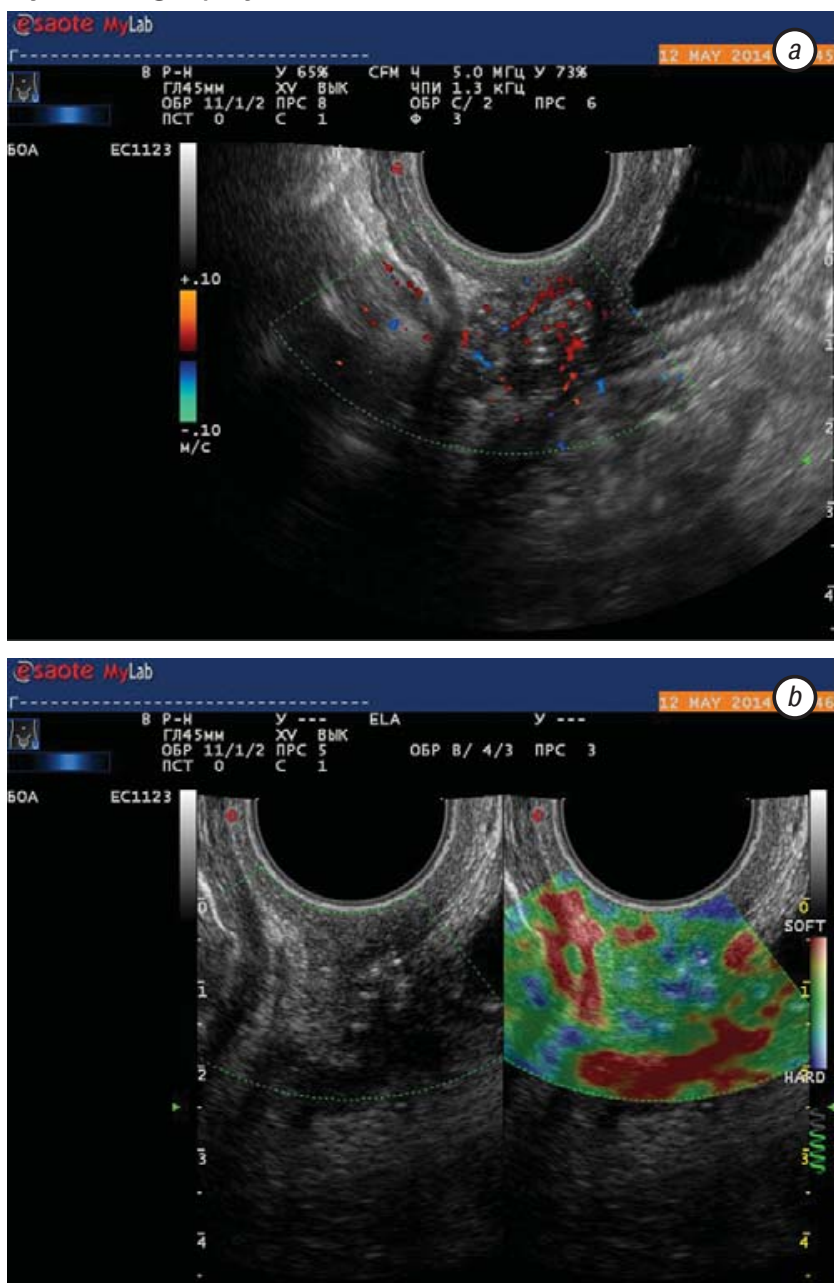

Fig. 6. The inflammatory infiltrate in the vaginal stump after 6 months of combination therapy. At the Doppler mode (a), increased blood flow is registered, at elastography $(b)$ formations have elastic nature

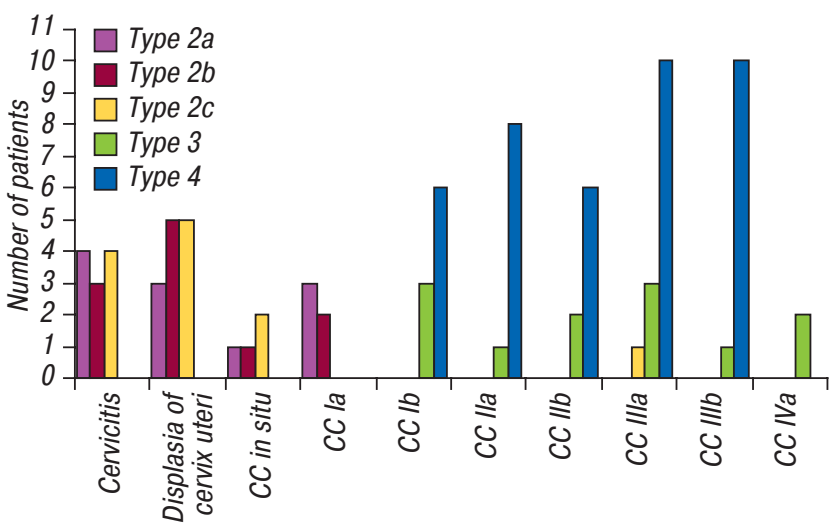

Fig. 7. Elastography type's distribution at patients with cervix pathologies

Fig. 8 demonstrates elastogram types distribution during monitoring of the patients treated with conservative therapy: after treatment phase 1, after its finishing and with recurrent disease.

As can be seen from the diagram, just after stage 1 of the chemoradiotherapy distribution of elastogram types shifted (in comparison with start of treat- ment) towards less severe. Elastogram type 3 became more common (50\% of cases), type 4 characteristic for malignant tumors was determined rarer than before treatment (40\%). After the treatment finishing elastograms were similar to that in non-malignant processes with dominated elastic types. But in $25 \%$ of cases we met the stiff type 3 , reflecting the large number of rigid post-radiation fibrous inclusions in the cervix. In recurrent CC distribution of elastographic types was similar to that seen in the primary tumor.

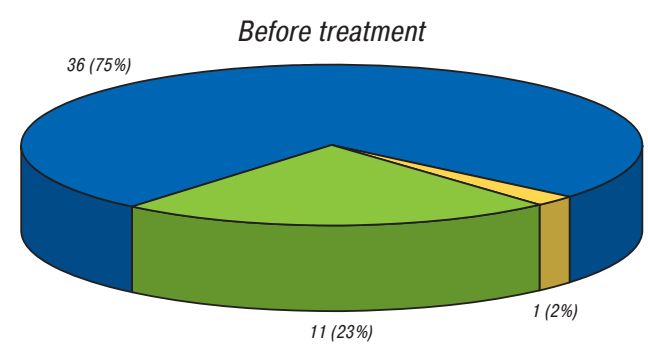

After treatment phase 1
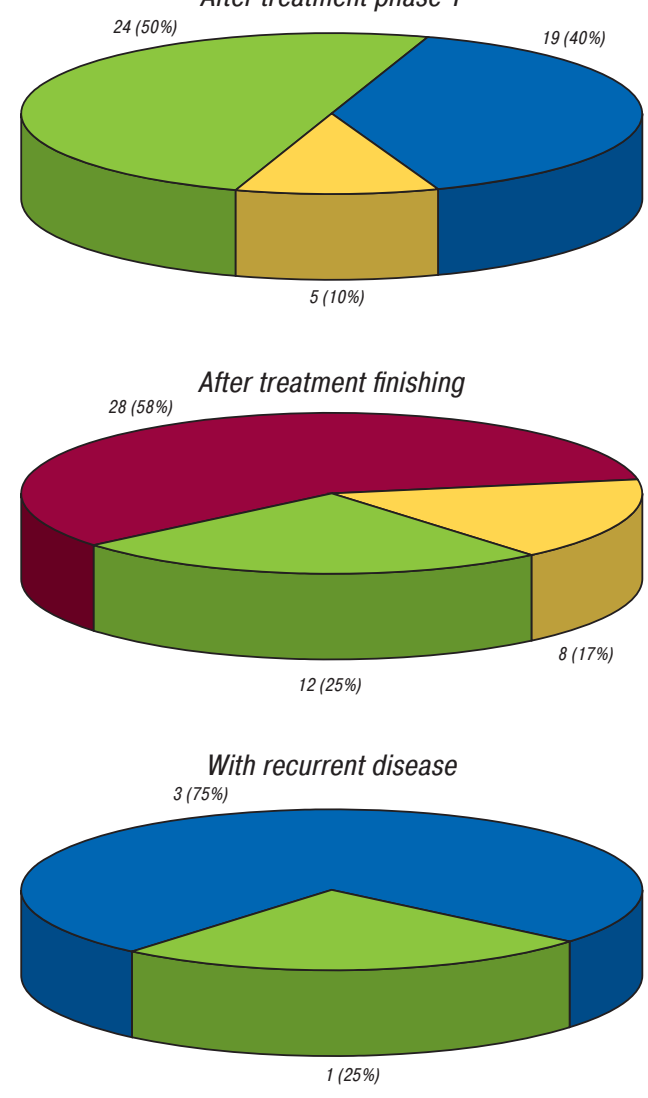

$\square$ Type 2b $\square$ Type 2c $\square$ Type $3 \quad \square$ Type 4

Fig. 8. Elastography types distribution during chemioradiotherapy effectiveness monitoring

Our studies have also demonstrated the benefits of elastography in the evaluation of tumor process invasion which improved the accuracy of CC stages determination and was of great importance for treatment planning.

Table 2 presents diagnostic value indices of "US" and "US + EG" systems for estimation cancer invasion into the parametrium, uterus body and vagina. In patients after operations verification of ultrasound investigation was performed on the basis of surgical intervention data and pathology analysis, MRI data was used as the reference method for the rest of the patients. 
Table 2. Diagnostic value indices of "US" and "US + EG" systems for estimation cancer invasion at CC

\begin{tabular}{lcccccc}
\hline Detection of tumor & \multicolumn{2}{c}{ Sensitivity, \% } & \multicolumn{2}{c}{ Specificity, \% } & \multicolumn{2}{c}{ Accuracy, \% } \\
\cline { 2 - 7 } \multicolumn{1}{c}{ invasion } & US & US + EG & US & US + EG & US & US + EG \\
\hline Into the parametrium & 77.1 & 91.4 & 85.1 & 96.2 & 80.6 & 93.5 \\
Into vagina & 75.0 & 83.3 & 76.9 & 88.4 & 75.8 & 85.4 \\
Into uterus body & 82.9 & 85.3 & 85.7 & 95.2 & 83.8 & 88.7 \\
\hline
\end{tabular}

Note: US - ultrasound diagnostics; EG - elastography.

As can be seen from the table 2, the use of elastography in the diagnostics has been given us an advantage in estimation CC invasion processes in comparison with standard ultrasound examination, which allowed to increase this methods informativeness to MRI indices levels. The use of elastography investigation profited most (according to all indices) for the assessment of tumor invasion: sensitivity, specificity, and accuracy of the detection increased 14.3; 11.1 and $12.9 \%$, respectively.

Elastography, like any other method has its strengths and weaknesses as soon as in specific application areas. Elastography limiting factor is its low informativeness at detection of CC early stages. It is difficult to differentiate tumor invasions of very small sizes from fibrous inclusions, also having a high rigidity. It should be emphasized that elastography differentiates elastic formations from stiff ones but not non-malignant from malignant. Also, limiting is the depth of the formations localization and their mobility. Elastography profits most for the diagnosis of inactive formations, localised at a depth of 0.5 to $5 \mathrm{~cm}$.

\section{CONCLUSIONS}

Thus, our investigations demonstrated that the sonoelastography inclusion into the complex ultrasound studies extended the diagnostic possibilities of these methods, allowed to increase the informativeness at estimation of cancer process stages (at the detection of tumor invasion into the parametrium sensitivity increased from 77.1 to $91.4 \%$, specificity of 85.1 to $96.2 \%$, accuracy - from 80.6 to $93.5 \%$; into the vagina: from 75.0 to $83.3 \%$, from 76.9 to $88.4 \%$, from 75.8 to $85.4 \%$, respectively; into the uterus body: from 82.9 to $85.3 \%$, from 85.7 to $95,2 \%$, from 83.8 to $88.7 \%$, respectively). Its use in complex with other methods of investigation may improve diagnostic quality at threatening pathologies such as $\mathrm{CC}$. This elastography method is inexpensive, simple in use, does not occupy plenty of time in the routine study, non-invasive, safe. Currently elastography technique is in the process of development and requires further study.

\section{REFERENCES}

1. Botar JC, Vasilescu D, Damian L. Musculoskeletal sonoelastography. Pictorial essay. Med Ultrason 2012; 14: 239-45.

2. Adamietz BR, Meier-Meitinger M, Fasching P, et al. New diagnostic criteria in real-time elastography for the assessment of breast lesions. Ultraschall Med 2011; 32: 67-73.

3. Bota S, Sporea I, Sirli R, et al. Intra- and interoperator reproducibility of acoustic radiation force impulse (ARFI) elastography preliminary results. Ultrasound Med Biol 2012; 38: $1103-8$.

4. Barr RG, Destounis S, Lackey LB, et al. Evaluation of breast lesions using sonographic elasticity imaging: a multicenter trial. J Ultrasound Med 2012; 31: 281-7.

5. Arda K, Ciledag N, Aktas E. Quantitative assessment of normal soft-tissue elasticity using shear-wave ultrasound elastography. Am J Roentgen 2011; 197: 532-6.

6. Swiatkowska-Freund M, Preis K. New methods of ultrasound examination of uterine cervix before labor induction. Gin Pol Med Project 2010; 3: 9-15.

7. Swiatkowska-Freund M, Preis K. Elastography of the uterine cervix: implications for success of induction of labor. Ultrasound Obstet Gynecol 2011; 38: 52-6

8. Molina F, Gomez L, Florido J. Quantification of cervical elastography. A reproducibility study. Ultrasound Obstet Gynecol 2012; 39: 685-9.

9. Gazhonova V, Churkina S, Lukyanova E, et al. Clinical application of new method - sonoelastography in gynaecology. Kremlin medicine. Clinical Herald 2008; 2: 1823 (in Russian).

10. Van den Bosch T, Coosemans A, Morina M. Screening for uterine tumours. Best Pract Res Clin Obstet Gynaecol 2012; 26: 257-66.

11. Balleyguier C, Sala E, Da Cunha T, et al. Staging of uterine cervical cancer with MRI: guidelines of the European Society of Urogenital Radiology. Eur Radiol 2011; 21: 1102-10.

12. Mitchell DG, Snyder B, Coakley F, et al. Early invasive cervical cancer: MRI and CT preditors of lymphatic metastases in the ACRIN 6651/GOG 183 intergroup study. Gynecol Oncol 2009; 112: 95-103.

13. Kesic V. Management of cervical cancer. Eur J Surg Oncol 2006; 32: 832-7.

14. Maneo A, Colombo A, Landoni F, et al. Treatment of stage IIIB cervical carcinoma. A comparison between radiotherapy, concurrent chemo-radiotherapy and neoadjuvant chemotherapy. Minerva Ginecol 2005; 57: 141-52.

15. Houvenaeghel G, Lelievre L, Gonzague-Casabianca L, et al. Long-term survival after concomitant chemoradiotherapy prior to surgery in advanced cervical carcinoma. Gynecol Oncol 2006; 100: 338-43.

16. Akbayir O, Corbacioglu A, Numanoglu C. Preoperative assessment of myometrial and cervical invasion in endometrial carcinoma by transvaginal ultrasound. Gynecol Oncol 2011; 122: $600-3$. 